\title{
Concepts for the Construction of a New National Drug Service System
}

\author{
Wang Jian $^{1}$, Yuan Chunping ${ }^{1}$, Wang Hao ${ }^{1}$, Zhang Zhirong ${ }^{2}$, Zhang qiang ${ }^{3}$, Chen Guiliang ${ }^{4}$, Liu Changxiao ${ }^{5}$, \\ Hou Huimin ${ }^{1}$, Yang Shengli ${ }^{6}$
}

1. National Pharmaceutical Engineering Research Center, Shanghai 201203, China

2. West China School of Pharmacy, Sichuan University, Chengdu 610041, China

3. School of Pharmaceutical Sciences, Peking University, 100191, China

4. Shanghai Institute for Food and Drug Control, shanghai 201203, China

5. Tianjin Institute of Pharmaceutical Research, Tianjin 300193, China

6. Shanghai Institutes for Biological Sciences, Chinese Academy of Sciences, Shanghai 200031, China

\begin{abstract}
Although the current drug service system can meet the fundamental healthcare needs of China, outstanding issues exist in all service sectors, including research and development, manufacturing, marketing, and clinical use. Through a literature search, data queries, specialist consultation, and a panel discussion, this study investigated the current status of the drug service system and identified the main problems. We propose an idea for constructing a new national drug service system to provide drugs to the Chinese people that are of high quality, have a reliable therapeutic effect, come at a reasonable price, and exhibit good medical compliance, thus ensuring the efficacy, safety, availability, and reasonability of drugs for clinical use, as well as promoting a structural transformation of the pharmaceutical industry that will enable China to finally transition from a big country into a great power in the global pharmaceutical industry.
\end{abstract}

Keywords: drug; service system; pharmaceutical industry; supervision; development strategy

\section{Introduction}

Drugs are special commodities and strategic materials, which are related to people's health, social harmony, and national security, and the government has paid much attention to them, while widespread concern has been expressed across society as a whole [1]. With decades of development, a drug service system consisting of different functional groups such as research and development, manufacturing, marketing, clinical use, and supervision, has been established and developed rapidly in China. From the synthesis of insulin and discovery of artemisinin, to the large-scale production and export of antibiotics and other bulk drug materials, as well as the successful development of SARS and Ebola vaccines, all of these indicate the growth and progress of the pharmaceutical industry of China.

To boost the rapid economic development since China's reform and opening to the outside world, the admittance threshold for the pharmaceutical industry was lowered for drug research and development, manufacturing, and distribution. Private capital investments in pharmaceutical enterprises increased rapidly and became an important component of drug service systems. Meanwhile, attracted by the reform and opening policy and the huge market demand, internationally renowned pharmaceutical enterprises have also set up manufacturing plants and R\&D centers in China. Therefore, the pharmaceutical industry presents diversified economic characteristics, further promoting the

Received date: January 10, 2017; Revised date: March 1, 2017

Corresponding author: Yang Shengli, Shanghai Institutes for Biological Science, Chinese Academy of Sciences, Professor; Chinese Academy of Engineering, Academician. Major research field is biotechnological drugs. E-mail: slyang@srcb.ac.cn

Funding program: CAE Advisory Project "Development Strategy for National Health Promotion and Medical and Healthcare Undertakings in China" (2014-ZD-06) Chinese version: Strategic Study of CAE 2017, 19 (2): 062-067

Cited item: Wang Jian et al. Concepts for the Construction of a New National Drug Service System. Strategic Study of CAE, https://doi.org/10.15302/ J-SSCAE-2017.02.010 
development of China's pharmaceutical industry. As a strategic new industry, the growth rate of the pharmaceutical industry has been at the forefront of Chinese economic development since the 11th Five-Year Plan. As a result, China has become the global manufacturing base for bulk drugs and a genuinely big country in the pharmaceutical industry, and played an extremely important role in ensuring our public health.

However, with the fast development of the pharmaceutical industry, prominent problems have also emerged in the drug service system, which cannot fully satisfy fast-growing health needs and sustainable pharmaceutical industry development [1]. Therefore, based on the investigation and analysis of problems in the existing drug service system, the concept of building a novel national drug service system was proposed, so as to ensure the quality and supply of drugs as strategic materials, guarantee the effectiveness, safety, accessibility, and rationality of clinical application, satisfy the people's health needs and the sustained and healthy development of Chinese pharmaceutical industry, and promote transformation from a big pharmaceutical country into a global pharmaceutical power.

\section{The present situation of the Chinese drug service system}

\subsection{The quality and supply of drugs can meet the fundamental needs of clinical medication}

By the end of 2015, there were about 5000 pharmaceutical manufacturing enterprises, 26000 good manufacturing practice (GMP) certificates issued, and 16000 commercial drugs with 187000 approval numbers, of which about 7000 are chemical drugs with 121000 approval numbers [2]. There were also 140000 drug distributors, 430000 retail drugstores, and 970000 medical institutions. The 2010 edition of GMP standards was close to EU requirements, and the 2015 edition of the Chinese Pharmacopoeia was close to United States Pharmacopoeia (USP) standards. In recent years, the functions and responsibilities of drug regulatory agencies and quality control institutions at national, provincial, municipal, and county levels have been improved, and drug registration, review, and approval systems have been reformed. Additionally, the national essential drug system has been implemented. Overall, the drug quality and supply have been able to meet the basic needs of medication and healthcare in China.

\subsection{R\&D input and scientific achievements increased significantly}

In 2015, the total R\&D expenditure of pharmaceutical enterprises in China amounted to 45 billion yuan, which quadrupled that of 2010 [2]. With the strong support and promotion of the National Major Scientific and Technological Special Project for
Significant New Drugs Development during the 12th Five-Year Plan period, a number of high-quality innovation achievements have sprung up. A total of 210 innovative drugs were approved for clinical research, 15 innovative drugs of state category I were approved for production, and more than 110 generic drugs received market approval. The quality control technology for traditional Chinese medicine has been greatly improved, and a significant milestone was when pharmacy expert Tu Youyou won the 2016 Nobel Prize in physiology and medicine. In the 2016 National Science and Technology Awards Conference, a total of 39 projects in the field of medicine and healthcare were awarded the national Science and Technology Awards, 14 of which were achievements of the two National Major Scientific and Technological Special Projects led by the National Health and Family Planning Commission.

\subsection{The growth rate of the pharmaceutical industry ranked first in all industries and began to move towards the international community}

As a new strategic industry, China's pharmaceutical industry has developed rapidly. During the period of the 11th Five-Year Plan, the total output value of the pharmaceutical industry grew by an average annual rate of over $23 \%$. During the period of the 12th Five-Year Plan, the gross pharmaceutical industrial output value jumped to the second place in the world, only next to that of the United States of America. The industrial added value of pharmaceutical enterprises increased by an average annual rate of $13.4 \%$, accounting for the proportion of industrial added value that increased from $2.3 \%$ to $3.0 \%$. The average growth rates of main business income and total profit were $17.4 \%$ and $14.5 \%$, respectively, ranking first in all industries. In 2015, the main business income of pharmaceutical enterprises was 2688.5 billion yuan with an increase of $9.5 \%$ compared with the previous year, and the total profit was 276.8 billion with an increase of $12.5 \%$.

With the rapid growth of scale benefits, drug varieties were becoming increasingly abundant. The commonly used chemical and biological drugs could be manufactured domestically. Bulk drug materials such as antibiotics could not only meet the needs of the domestic market but were also exported to developed countries as the world's largest supplier. A batch of advanced technologies such as biocatalysis, chiral synthesis, and new drug delivery systems (DDS) have been industrialized; antibody and other biotechnological drugs were developed rapidly; the R\&D levels of vaccines against SARS, avian influenza, hepatitis E, and Ebola have reached advanced international ranks.

In addition to meeting domestic demands, homemade drugs have begun to move towards the global market. In 2015, the total export value of the pharmaceutical industry was 56.4 billion USD [2], and the export structure has been improved as the proportion of preparations increased gradually. The sales of preparations exported to developed countries have also 
achieved a breakthrough. The number of enterprises planning to export preparations has been increasing, and overseas investment developed from the establishment of R\&D centers to the set-up of production bases. Additionally, drug research and development has gradually been geared to international standards and requirements. So far over 100 generic drugs have obtained registration approval from European and US authorities, and applications for clinical research to the FDA have been made for more than 50 new drugs.

\subsection{Regulatory reform continued to advance}

In recent years, the State Council and the China Food and Drug Administration (CFDA) issued a series of important documents on strengthening R\&D supervision, speeding up drug review and approval, improving drug quality, and perfecting drug price reform. For example, reform of drug prices was put forward, a pilot program of a "two invoice system" was launched, and a medical insurance fee control plan was proposed. The most rigorous self-examination and verification of the data from clinical trials were carried out, quality consistency evaluation of the generic drugs was initiated and training was carried out nationwide, the reform program for registration and classification of chemical drugs has been put into effect, and the concept of self-examination and verification of the drug manufacturing process has been put forward. The release and implementation of these policies will play a very important role in strengthening drug research and development, speeding up drug review and approval, improving drug quality, and standardizing drug manufacturing and distribution.

\section{Main problems of the drug service system in China}

\subsection{Drug quality has not yet reached an internationally advanced level}

For various reasons, there were some defects in the quality standards of certain domestic drugs. The quality of some generic chemical drugs in the market is inconsistent with the original ones, and some drugs did not meet the requirements of relevant standards. Therefore, overall drug quality did not reach an internationally advanced level, resulting in distrust by many medical staff and patients of the quality of domestic drugs [3]. In 2012, the quality consistency evaluation of generic chemical drug was brought into the State Council's National 12th Five-Year Plan for Drug Safety, but progress was extremely slow [4].

\subsection{The coexistence of high drug prices and false low drug prices}

Because of irregularities in drug distribution and marketing, loopholes in the centralized bidding system, and vicious competition among the manufacturing enterprises, virtually high pricing, rebate promotions, and even bribe promotions have become the mainstream means of drug marketing. The higher the drug price, the easier it was to sell, which led to virtually high drug prices. For some drugs, the retail price was dozens or even hundreds of times of the factory price. The situation of high drug prices was also quite prominent in foreign capital enterprises in China, which had the sole pricing power during the previous compulsory price reductions and maintained the high drug prices.

At the same time, due to compulsory price reductions or restrictions on essential drugs during the past decades at the command of the National Development and Reform Commission, and because some manufacturing enterprises took a "predatory pricing" strategy in order to obtain centralized bidding targets, the price of some essential drugs was set close to or even below the average cost, resulting in false low drug prices and finally market shortages.

By the end of 2014, the National Development and Reform Commission issued a draft document to provincial price control agencies, suggesting the release of drug price controls for essential drugs. In June 2015, the Opinions on Pushing Forward the Pharmaceutical Pricing Reform were officially implemented. However, due to the unreasonable drug distribution and marketing system, market-oriented drug price formation mechanisms could not be established in a day. The phenomenon of high drug prices and false low drug prices still existed, and shortages of some essential drugs have not yet been improved.

\subsection{Drug preparations for children and elders are in short supply and abuse is serious}

The prevalence rates of children and elders are higher than that of ordinary adults, and they may need different dosage forms from normal adults. For example, drug consumption in China for elders accounted for more than $50 \%$ of the total drug market, but there are a series of problems associated with the administration of ordinary preparations such as difficulty of swallowing solid dosage forms, forgetting to take medicines, inconvenience of repeated administration, and use of several kinds of drugs at the same time. However, the drug varieties, formulations, specifications, and packages suitable for children and elders are in short supply. In fact, clinical drug administration for these two types of patients was performed mainly by adjusting the dosage of common adult formulations according to experience. In addition, irrational drug use is prominent, including abuse of antibiotics, hormones, and vitamin supplements especially [5-7].

\subsection{Lack of innovative drugs and low utilization of advanced DDS}

New drug innovation ability, especially original innovation 
ability, was insufficient. There were few genuine innovative chemical drug products in the market, and more than $95 \%$ of them were generic drugs. Drug research and development did not fully conform to the rigorous standards laid down by the CFDA. Although more than 100 kinds of DDS have market approval from CFDA, many of them could not be repeatedly obtained from large scale production, and the quality was also questionable, with a total clinical utilization rate of less than $5 \%$, even much lower than that of India (more than 10\%). For Europe, the United States of America, and other developed countries, new DDS have been widely used, including oral controlled/sustained released DDS, transdermal DDS, new types of injections (liposomes, microspheres, drug-loaded lipid emulsions, micelles, nanoparticles, etc.), novel films, mucosal DDS, inhalation DDS, and so on, accounting for more than $20 \%$ of total clinical drug use.

3.5 The pharmaceutical enterprises were big in quantity but small in scale, the concentration ratio was extremely low, the industrial structure was irrational, and the pharmaceutical equipment and processing technology were not updated

3.5.1 The pharmaceutical enterprises were big in quantity but small in scale and the market concentration ratio (CR) was extremely low

In 2011, the CR4 and CR8 (the market share of the top 4 and top 8 pharmaceutical enterprises in the total market) were $7.6 \%$ and $11.4 \%$, respectively, and the total market share of the top 10 enterprises (CR10) was less than $15 \%$, indicating a very low concentration ratio of the pharmaceutical industry. In the same year the CR10 of the US pharmaceutical industry was $63 \%$. Among the statistics for pharmaceutical enterprises in 2012, large enterprises only accounted for $3.5 \%$, medium-sized enterprises accounted for $18.6 \%$, and small enterprises accounted for $77.9 \%$. In 2013, the sales income of the top 100 pharmaceutical enterprises accounted for only $28.8 \%$ of the total sales revenue of this industry. The total main business revenue of the chemical drug preparation industry was 573.1 billion yuan in China, while the main business revenue of Johnson \& Johnson alone was 71.31 billion USD (about 439.2 billion yuan) [8].

Low concentration ratio is neither good for regulation nor for innovation. There were more than 100 or even 500 factories manufacturing the same drugs and preparations, which inevitably led to vicious competition, most commonly using the means of "rebate." Pharmaceutical enterprises are great in number, small in scale, and scattered in fields, leading to lack of integration of industrial resources and low efficiency, which is not conducive for enterprises to strengthen technological innovation capability and market competitive power as well.

3.5.2 Irrational industrial structure and slow internationalization steps

Although, internationally, China is a large pharmaceutical country, the structure of the pharmaceutical industry is irrational. On the one hand, large quantities of low added value raw drug materials and intermediates were exported and pollution was left in China, while on the other hand, large amounts of drug preparations, especially advanced DDS with high attachment value, were imported and the profits left abroad. With the ever-increasing requirement of environmental protection and production costs, the implementation of medical insurance fee controls and increasingly rigorous drug regulatory policies, the overall growth rate of the pharmaceutical industry has slowed down and a difficult structural transition period has been coming.

In 2015, China became the second largest drug market in the world, but the international competitive capacity of the domestic pharmaceutical products was very low. The World Health Statistics 2009 Yearbook of the Organization showed that the number of domestic drugs that entered the procurement catalog of World Health Organization was 6, while for India it was 194. From 2011 to 2013, the FDA approved 19 Chinese generic drugs and 476 Indian generic drugs, and the total approval number was 1307.

3.5.3 The pharmaceutical equipment and manufacturing technology have not been updated

In the pharmaceutical packaging area, highly automated equipment has been introduced from abroad in recent years, and some has been made domestically. Advanced instruments and equipment for pharmaceutical analysis and inspection have been imported too. However, the pharmaceutical equipment and manufacturing technology were backward in general, and even significantly lag behind the food manufacturing industry. In fact, information technology and artificial intelligence have been widely used in the manufacturing industry. Process analysis technology (PAT), intelligent manufacturing, continuous manufacturing, and other new technologies have been widely applied in the food, tobacco, automobile, and other industries, but much less in the pharmaceutical preparation industry. Drug manufacturing still relied on manual operation to a great extent At the same time, the manufacturing management level was not updated, and information and network technology have not yet been applied to the production management system.

\subsection{Slow drug review and approval and lack of scientific supervision patterns and techniques}

\subsubsection{Slow drug review and approval}

In previous years, the CFDA did not have enough drug reviewers to complete the large number of registration applications, resulting in a great backlog of applications. Some urgently needed drugs could not be reviewed and approved, and the R\&D achievements could not be converted into products in time and put to use. Since the drug review and approval reform in 2015, review efficiency has been substantially enhanced. A total of 
9601 registration applications were reviewed throughout the year, 1390 copies more than the amount received this year, but the total backlog was still great.

\subsubsection{Lack of scientific supervision patterns and techniques}

Disorderly or even fraudulent behavior such as adulteration and data falsification existed in many pharmaceutical service sectors, including R\&D, manufacturing, distribution, and clinical application; therefore, the service integrity in the pharmaceutical industry was questionable. Due to lack of scientific and effective means and techniques, the supervision mainly relied on onsite verification and flight inspection, which was not only timeconsuming but also had difficulty in finding problems. The penalties for misbehavior and disorderly activities were not enough and could not have a deterrent effect.

3.6.3 The regulatory authorities failed to lead drug innovation and industry development

As the supervisors and leaders of the pharmaceutical industry, regulatory authorities should not only be able to discover and solve drug quality and safety problems, but also be able to lead drug innovation and industrial development. The regulatory authorities have neither established effective channels of communication with pharmaceutical enterprises and $R \& D$ institutions, nor provided practical leadership and guidance to drug innovation research and industrial development.

For example, drugs that have not yet been approved by the FDA or other regulatory authorities of developed countries are quite difficult to obtain approval for in China. Again, in the past two years, the CFDA have approved a large number of clinical trials, and quality consistency evaluation of generic drugs has been officially launched. However, there were only more than one hundred clinical pharmacological research bases in China with phase I clinical trial qualification (including BE research), the resources for BE research were extremely insufficient, and prices have also risen sharply, bringing enormous pressure to pharmaceutical enterprises. Regulatory authorities should formulate relevant policies as soon as possible to solve this problem.

\section{Development strategy of a new national drug service system}

\subsection{Overall objectives}

To achieve a real-time and effective supervision of all aspects of our pharmaceutical industry including drug R\&D, review and approval, manufacturing, distribution, and clinical use, a novel national drug service system was proposed, so as to comprehensively improve the quality of domestic drugs to an internationally advanced level, standardize drug distribution and marketing, and rationalize the clinical application, and therefore provide domestic drugs with high quality, reliable therapeutic effects, reasonable prices, and good medical compliance. At the same time, pharmaceutical preparations suitable for special patients and advanced DDS should be developed with great effort. The technology of intelligent and continuous manufacturing should be vigorously exploited to completely improve the level of the pharmaceutical industry, put domestic medicines forward to the international market, promote the structural transformation and sustainable development of the pharmaceutical industry, and finally transition from a big pharmaceutical country into a great power of the global pharmaceutical industry.

\subsection{Construction of a new national drug service system}

As different drug service sectors in China belonged to different ministries and commissions, the management of the pharmaceutical industry in the past was fragmented, and the pharmaceutical information, resources, and policies have not been centralized and unified. Through construction of a novel national drug service system, information sharing, resource integration, unified decision-making, and scientific supervision can be realized, so as to meet ever-growing and multi-level health needs and to promote sustainable development of the pharmaceutical industry.

4.2.1 Construction of a national drug service system based on "Internet Plus"

The information and internet based national drug supervision center and drug service system should be built with the Ministry of Industry and Information Technology, National Health and Family Planning Commission, and the CFDA as the regulation subjects, with drug R\&D, manufacturing, distribution, marketing, and medical institutions as the service subjects, and with the patients as the service objects using advanced information and network technology, so that all the related drug information of the service subjects can be achieved from the supervision system whenever needed, intelligent supervision of all aspects of drug services will be realized, various types of on-site inspections and flight inspections shall be exempted, drug distribution and marketing order will be regulated, and drug safety, effectiveness, availability, and rationality of clinical medication will be ensured.

The fifth article of the Drug Administration Law of the PRC (2015 Revision) specified that the drug supervision and administration agency of the State Council is in charge of the supervision and management of drugs throughout the country. Therefore, in the novel national drug service system, CFDA should not only play the role of drug quality control, but also exert the function of industrial services, innovation leadership, and development guidance.

\subsubsection{Speed up quality revaluation of commercial drugs}

Through quality revaluation of commercial drugs, the quality of domestic drugs should be entirely improved to reach an internationally advanced level. Quality assessment of national essen- 
tial drugs should be completed during the 13th Five-Year Plan period; revaluation of advanced DDS should be finished during the 14th Five-Year Plan period, mainly including controlledrelease preparations, delayed release preparations, enteric preparations, transdermal preparations, novel injections, mucosal preparations, inhalations preparations, and non-injection formulations for biotechnology drugs (polypeptides, proteins, and vaccines); revaluation of other drugs should be finished during the 15th Five-Year Plan period, fully reaching the quality of drugs of the developed countries, and finally realizing the dream of a strong pharmaceutical country.

4.2.3 Develop advanced DDS and preparations for special populations, and promote large-scale production as well as wide clinical application

(1) It is recommended to implement FDA classification and registration management procedures for chemical drugs, encouraging the development of diversified dosage forms to meet the needs of different populations, reduce costs, achieve green manufacturing, reduce energy consumption, and so on, all of which should be supported.

(2) Vigorously develop advanced generic drugs to improve therapeutic effects, reduce side-effects and improve patients' compliance, as well as reduce the total cost of medication and save medical insurance resources.

(3) Develop pharmaceutical preparations for special populations such as children and elders, as well as preparations for strategic reserves including drugs for infectious diseases and emergency situations.

(4) Promote the research and development of intelligent DDS and technologies, including micro-electro- mechanical system (MEMS) drugs, active targeting DDS, intelligent and/or environmental sensitive DDS, wearable DDS or devices, functional pharmaceutical excipients, combination systems of drugs and devices, intelligent drug packages, and so on, so as to maximize the therapeutic effects of drugs and patients' compliance.

\subsubsection{Enhance construction of innovative capability}

Regulatory and market mechanisms for encouraging development and application of innovative drugs should be established. An innovative pattern with enterprises as the main body and combination of industry-university-institute should be formed, enhancing R\&D innovation capability. At the same time, urgently needed innovative drugs should be included in medical insurance management so as to promote clinical applications.

4.2.5 Establish a research center for intelligent and continuous drug manufacturing and promote development of the pharmaceutical industry

A research center for intelligent and continuous drug manufacturing should be established with the application of new technologies such as process analysis, automation, information, and artificial intelligence. The development of advanced DDS and corresponding manufacturing equipment should be emphasized, including continuous manufacturing technology and equipment, hot melt extrusion technology and equipment, micro/nano spray technology and equipment, 3D printing technology and equipment, and so on. Intelligent pharmaceutical manufacturing management systems should also be developed to achieve intelligent monitoring of the quality of raw materials, manufacturing process, packaging, as well as the quality of semi-products and finished products, fundamentally guaranteeing the quality of drugs.

4.2.6 Promote the merger and acquisition of pharmaceutical enterprises to improve the concentration ratio and ensure healthy development of the pharmaceutical industry

Relevant policies and measures should be put forward, leading and guiding enterprises to carry out mergers and acquisition according to market rules, improving the concentration ratio and competitiveness of the pharmaceutical industry, realizing the transformation of industrial structure, and finally promoting the sustainable and healthy development of the pharmaceutical industry.

\section{References}

[1] Fan D M. Health care reform: Addition, subtraction, multiplication and division [J]. Negative, 2016, 7(3): 1-20. Chinese.

[2] Ministry of Industry and Information Technology of the People's Republic of China, National Development and Reform Commission of the People's Republic of China, Ministry of Science and Technology of the People's Republic of China, Ministry of Commerce of the People's Republic of China, National Health and Family Planning Commission of the People's Republic of China, China Food and Drug Administration of the People's Republic of China. Notice on issuing guide for development of pharmaceutical industry [EB/OL]. (2016-10-16) [2016-11-07]. http://www.miit. gov. cn /n1146295/n1146592/n3917132/n4061512/c5343399/content. html. Chinese.

[3] Yang M C, Wang L B, Lin M, et al. Research on analysis of quality difference and risk control between domestic and imported drugs [J]. Shanghai Food and Drug Information Research, 2011, 12(6): 33-45. Chinese.

[4] Drug Registration Division of China Food and Drug Administration of the People's Republic of China. Drafting instruction of the work plan for evaluation of the quality consistency of generic drugs (Draft) [EB/OL]. (2012-11-22) [2016-11-15]. http://www. sda.gov.cn /wso1/CL0778/76295.html. Chinese.

[5] Wang X L, Guo C Y. Analysis of present situation of pediatric drug formulation and specifications in foreign countries [J]. Chinese Pharmaceutical Journal, 2013, 48(17): 1317-1320. Chinese.

[6] Chen Q, Du G. The present situation and the management strategy of pediatric drug dosage forms [J]. Journal of Pediatric Pharmacy, 2013, 19(8): 49-52. Chinese. 
[7] Delgado-Charro M B, Guy R H. Effective use of transdermal drug delivery in children [J]. Advanced Drug Delivery Reviews, 2014, 73: 63-82.
[8] Kuang L C, Feng G Z. Analysis on the development status of Chinese pharmaceutical industry [J]. Modern Business Trade Industry, 2015(10): 7-9. Chinese. 